Relations industrielles

Industrial Relations

\title{
La survie de droits subjectifs à la convention collective
}

\section{Fernand Morin}

Volume 40, numéro 4, 1985

URI : https://id.erudit.org/iderudit/051381ar

DOI : https://doi.org/10.7202/051381ar

Aller au sommaire du numéro

Éditeur(s)

Département des relations industrielles de l'Université Laval

ISSN

0034-379X (imprimé)

1703-8138 (numérique)

Découvrir la revue

Citer cet article

Morin, F. (1985). La survie de droits subjectifs à la convention collective. Relations industrielles / Industrial Relations, 40(4), 847-855.

https://doi.org/10.7202/051381ar

Tous droits réservés @ C Département des relations industrielles de l'Universite Laval, 1985
Ce document est protégé par la loi sur le droit d'auteur. L’utilisation des services d'Érudit (y compris la reproduction) est assujettie à sa politique d'utilisation que vous pouvez consulter en ligne.

https://apropos.erudit.org/fr/usagers/politique-dutilisation/ 


\section{La survie de droits subjectifs a la convention collective}

\section{Fernand Morin}

Face à la délicate question du rapport entre la convention collective et le contrat individuel de travail, la Cour d'appel prit une position assez particulière que nous proposons de souligner et d'analyser. Il s'agissait de savoir si deux contremaîtres susceptibles de réintégrer les rangs des salariés disposaient encore du même droit d'ancienneté prévalant au moment de leur nomination nonobstant les dispositions de l'actuelle convention collective. Le plus haut tribunal du Québec décida que ces dispositions de la convention collective en vigueur au moment de leur nomination devaient encore s'appliquer advenant le retour de ces contremaîtres à titre de salarié1. Nous tentons d'abord de rapporter aussi fidelement que possible les motifs retenus par la Cour d'appel au soutien de cette conclusion, ce qui devrait nous permettre par la suite de mieux la critiquer.

\section{LES FAITS}

Les données factuelles sont relativement simples et ne font pas l'objet de débat entre les interlocuteurs. Le résumé qu'en donna la Cour supérieure fut d'ailleurs repris à la Cour d'appel:

Au moment où les requérants faisaient partie de l'unité de négociation et étaient membres du syndicat mis en cause, de même que lors de leur promotion comme contremaître de l'intimé, la convention collective qui régissait les conditions de travail des parties comportait une clause d'ancienneté ordinaire, sans limitation. (p. 63)

Les deux conventions collectives postérieures à la nomination des plaignants à titre de contremaître modifièrent les règles du maintien de l'ancienneté en pareilles situations. Ainsi, la convention collective à l'étude, soit celle au moment où la Cour supérieure fut saisie de cette affaire, comprenait ses réserves et limites:

L'ancienneté signifie la période de temps pendant laquelle une personne a été employée sans interruption par la Compagnie à l'intérieur de cette unité de négociation et la période temporaire de 23 semaines de calendrier total par année de convention qu'une personne est promue hors de l'unité, telle que prévu à la clause 59 , sur une classification dans un ou plusieurs départements ainsi que les périodes qu'un employé promu hors de l'unité peut faire dans les éventuels retours à l'intérieur de l'unité. (p. 63)

- MORIN, F., professeur, Département des relations industrielles, Université Laval.

1 Syndicat national de l'amiante d'Asbestos Inc. c. Nadeau et al., 1985, CA 62.

Relat. Ind., vol. 40, no 4, 1985 ( ) PUL ISSN 0034-379 X 
Inquiets de la portée que pourrait éventuellement avoir cette disposition lors d'une réintégration toujours possible dans les rangs des salariés, ces deux contremaîtres demandèrent à la cour, par voie d'un jugement déclaratoire, de préciser quelle était la portée de cette dernière disposition limitative à leur égard.

\section{L'ARRÊT}

L'unanimité des trois juges ayant ententu cette affaire en appel et la complémentarité de leurs notes nous autorisent à souligner simultanément l'approche retenue. En premier lieu, la Cour d'appel convient qu'un tel jugement déclaratoire constituait un bon moyen d'intervention dans les circonstances et que ces contremaîtres ne devaient attendre que le problème pratique soit posé: «...that the potential problem is of sufficient importance to have it decided immediately» (juge Kaufman). M. le juge Vallerand dira même:

...qu'il serait d'un juridisme tout aussi sot que désuet d'imposer aux parties d'attendre l'inévitable difficulté immédiate pour s'adresser à la Cour supérieure sur le sujet de l'applicabilité de l'une ou l'autre des deux conventions et, cela fait de s'en aller chez l'arbitre de cette convention qu'aurait retenu la Cour. Sot, désuet et singulièrement contraire à l'esprit qu'anime la loi qui a inventé l'arbitrage des griefs précisément pour régler sommairement les conflits de travail. (p. 66)

Tout comme le juge de la Cour supérieure, la Cour d'appel justifie son intervention en soutenant qu'il ne s'agissait pas d'une question d'interprétation ni d'application d'une disposition de la convention collective, mais de son applicabilité. On s'appuie en cela sur la décision de la Cour suprême du Canada $^{2}$. Monsieur le juge Vallerand propose cette explication:

... supposons que les intimés, plutôt que de rechercher une déclaration de leurs droits éventuels (j'y reviendrai) avaient attendu que se manifeste un problème immédiat, par exemple des mises à pied selon l'ancienneté. Prenant bien sûr appui sur la première convention, ils se seraient adressés à un arbitre nommé par l'application de cette même convention et l'aurait prié d'en faire l'interprétation et l'application au cas. Mais voilà que l'employeur aurait été contraint d'introduire dans le débat la seconde convention qui, de l'avis du syndicat, fait échec à la première et, partant, d'inviter l'arbitre à interpréter et à appliquer celle-ci ce que, arbitre de la première convention, il n'aurait pas la compétence de faire. Il en irait, du reste, de même à l'inverse si le syndicat, sur la foi de la seconde convention avait entendu protester contre la reconnaissance aux intimés de l'ancienneté conforme à la première et donc, à son avis, contraire à la seconde. (p. 65)

\section{Ainsi, le juge Vallerand concluait qu'il devait s'agir}

... d'un cas d'applicabilité de litige préliminaire au sens de l'arrêt Labrecque que seule la Cour supérieure est compétente à résoudre à l'exclusion de l'un ou de l'autre des arbitres. (p. 66)

Ainsi, ces contremaîtres disposaient donc d'un droit:

2 P.G. Québec c. Labrecque (1980) 2RCS 1057. 
... However, good or desirable, it is, nevertheless, a right which is established by contract. And so it was in this case and the Respondents were entitled to rely on this. (juge Kaufman, p. 65)

Sur la question de fond qui consistait, selon cet entendement, à déterminer laquelle des deux conventions collectives pourrait s'appliquer, monsieur le juge Vallerand trancha ainsi:

Il n'y a pas, à mon sens, de contradiction entre les deux conventions sur le sujet qui nous retient. La première consacre le droit de ceux qui quittent l'unité pour devenir contremaîtres, le droit donc d'additionner l'ancienneté croissante pendant leur absence. Il s'agit ainsi d'un droit projeté exclusivement vers l'avenir et qui, par la force des choses, est, dès sa naissance, susceptible de se prolonger au-delà de la convention qui l'a créé et le soutient (...) (p. 66)

$\mathrm{Nul}$ ne contestera, en revanche, le principe qu'une convention collective lie non seulement ceux qui sont de l'unité lors de la signature mais tout autant ceux qui viendront par la suite. Dans cette optique les contremaîtres qui ne font pas partie d'une unité lors de la signature de la convention seront liés par celle-ci lorsqu'ils «reviendront» (...) ... «Le principe essentiel ici en cause ne paraît être celui de la non rétroactivité des dispositions, et singulièrement de celle qui ferait échec au droit par ailleurs acquis. Le droit de nos contremaîtres au respect de l'ancienneté assurée par la première convention est, je l'ai dit, un droit acquis dans le contexte de la seule premiere convention. (p. 66)

Monsieur le juge Chouinard retint également cette approche générale au motif principal que l'ancienneté:

... est au moins un fait corollaire au travail qui peut être source de droits, sinon un avantage personnel à l'employé qui seul pourrait en disposer. À ce sujet, plusieurs conventions collectives auront reconnu qu'un tel privilège d'ancienneté ne pouvait être négocié ni par l'employeur ni par un syndicat non accrédité à représenter le travailleur qui le possède. (p. 67)

\section{LE COMMENTAIRE}

Cette décision unanime illustre, une fois de plus, que le droit des rapports collectifs du travail ne s'impose pas encore avec autorité et aisance et, aussi, que les tribunaux veillent bien à limiter son hégémonie. Elle témoigne également d'une conception toute particulière du lien qui pourrait s'établir entre la convention collective et le contrat individuel de travail pour permettre la survie de droits qui résulte aux salariés d'une convention collective par ailleurs disparue. Parmi les nombreuses questions de droit que soulève cette affaire, nous en retenons trois: la notion d'applicabilité, la coexistence de droits résultant de conventions collectives successives et la notion d'ancienneté. Auparavant, signalons la sollicitude de la Cour à trancher cette question éventuelle des contremaîtres qui n'étaient pas encore rétrogradés ni mis à pied, tellement on considère l'ancienneté comme un droit personnel et quasi fonciamental. Selon le juge Vallerand ce serait «l'état syndical» individuel qui serait en cause. Il est vrai qu'il fallait dire qu'il en était ainsi, pour que la Cour supérieure et la Cour d'appel puissent s'autoriser à intervenir en pareille situation et par le biais d'un jugement déclaratoire. 


\section{L'applicabilité}

La Cour d'appel considère qu'il ne s'agirait pas, du moins à ce moment, d'une question relative à l'interprétation ni à l'application de la convention collective, mais strictement d'une question d'applicabilité d'une disposition conventionnelle. Est-ce là un nouveau moyen pour justifier l'intervention des tribunaux judiciaires en cette matière? Que faut-il entendre par l'applicabilité d'une disposition de la convention collective? Il s'agit, croyons-nous, d'évaluer la possibilité d'application d'une norme ce qui signifie, en termes plus claires et non jésuistiques, d'établir sa légalité, sa conformité à la loi et partant sa valeur contraignante. Tout autre considération que ce soit (la légitimité, l'opportunité ou la justesse d'une norme conventionnelle) ne pouvait être, à ce stade, du ressort de quelques instances judiciaires. Si la légalité d'une disposition conventionnelle était la véritable question posée, alors n'aurait-il pas fallu s'en tenir à cette triple question: par son sujet, son objet et sa fin, cette modalité conventionnelle est-elle conforme à la loi et à l'ordre public? Qu'en est-il? En premier lieu, soulignons que les plaignants n'étaient pas encore liés par cette disposition plus restrictive de la convention: l'article 67 du Code $d u$ travail énonce bien que seuls les salariés au sens de ce code y sont assujettis. Il est vrai qu'à ce même article, on traite du salarié futur, mais ne faut-il pas entendre ici le salarié qui le devient depuis la conclusion de la convention collective et non les futurs ou éventuels salariés? Quant à l'objet de cette disposition conventionnelle, l'ancienneté, n'est-elle pas manifestement reconnue à titre d'objet de négociation? Il est de commune connaissance que l'ancienneté constitue, dans la très grande majorité des conventions collectives, une condition de travail. L'article $62 C T$ reconnaît la pleine liberté des parties à négocier toutes conditions de travail non contraire à la loi ni à l'ordre public. Quelle serait donc la dispositon légale qui fut ainsi violée et en quoi l'ordre public en serait-il dérangé? La finalité de cette règle consiste d'abord à établir un ordre de priorité entre les salariés pour de multiples fins: promotion, choix de vacances, supplantation, mise a pied, réintégraton, etc. Outre que cette règle soit de pratique courante et qu'elle soit généralement acceptée entre les salariés, elle permet également de trancher facilement de longs débats entre le syndicat et l'employeur au sujet de l'exercice du pouvoir discrétionnaire de ce dernier. D'ailleurs l'ancienneté aussi est le critère facile, parfois trop il est vrai, pour désigner un porte-parole ou un porte-étendard d'un groupe, d'une communauté ou d'un collège même constitué de juges. On voit donc que sous cette triple voie, (sujet, objet et finalité) cette disposition conventionnelle pouvait difficilement être contestée. Or, il est manifeste que c'est nullement sous cet angle que la Cour d'appel aborda la question, mais bien sous celui de son applicabilité éventuelle c'est-à-dire dans l'hypothèse où ces deux contremaîtres reviendraient des salariés au sens de l'article 1 par. $1 \mathrm{du}$ Code du travail. Même sous cette dernière approche, la Cour considéra davantage la question de la survie de la disposition sur l'ancienneté de la convention collective expirée. Au soutien de sa décision, la Cour d'appel réfère à la décision de la Cour suprême du Canada dans l'affaire P.G. du Québec et Labrecque et al (1980) 2 RCS 1057). En ce dernier cas, la question principale posée consistait à déterminer si la Cour provinciale avait «... juridiction pour rendre ce jugement» (M. le juge Beetz) et subsidiairement, si Labrecque était assujetti à la convention collective ou, si l'on préfère, si 
elle lui était applicable. Avec respect pour l'opinion contraire, il nous semble que la question posée à la Cour d'appel était d'un tout autre ordre. $\mathrm{Ne}$ s'agissait-il pas de déterminer si une modalité (l'ancienneté) d'une convention collective par ailleurs expirée subsistait de quelque manière à l'arrivée d'une autre convention collective et au profit d'ex-salarié susceptibles de le redevenir? Selon notre entendement de la décision de la Cour d'appel, on considéra que ce droit à l'ancienneté accumulée était tellement collé à la personne des contremaîtres, leur «statut syndical», qu'il ne pouvait être altéré par la même source qui l'avait généré et sans le consentement exprès de deux contremaîtres. Cette logique impliquerait la coexistence, en un certain sens du moins, des deux conventions collectives.

\section{Deux conventions collectives}

La Cour d'appel n'affirma pas cette coexistence des deux conventions collectives. C'eut été d'ailleurs une hérésie trop flagrante. On semble cependant aborder la question comme s'il en était ainsi. Par exemple, lorsque le juge Vallerand tente de justifier cette intervention judiciaire, ne met-il pas en conflit les deux conventions collectives: «Prenant bien sûr appui sur la première convention...» (...) «... l'employeur a été contraint d'introduire dans le débat la seconde convention...» ou encore: "Sur la foi de leur convention (les contremaîtres) auprès de son arbitre qui appliquant sa convention...». Un telle argumentation n'exigerait-elle pas que l'on puisse écarter certains traits caractéristiques de toute convention collective conclue en vertu du Code? Nous n'en rappellerons que trois:

- une convention collective ne peut lier que les salariés de l'unité de négociation et ceux qui le deviennent au cours de son terme (art. 67 al. 1 $C t$;

- elle lie chacun des salariés de l'unité de négociation au-delà de leur volonté individuelle et le syndicat accrédité demeure responsable d'en surveiller l'application intégrale par tous et pour tous (art. 47.3, 69, 70, 100 et $100.3 \mathrm{Ct}$ );

- elle est unique (une seule à la fois pour un même groupe et en un même temps): art. 67 , al. $2 C t$ ) et nécessairement pour une durée limitée prédéterminée ou déterminable (art. 52.2 in fine, 65 et $66 C t$ ).

Ces seuls éléments permettent cependant de dégager quelques observations fort utiles à l'analyse de cette décision de la Cour d'appel:

- le salarié bénéficie des droits qui lui résultent d'une convention collective, mais dans la mesure où il est salarié (l'antécédent);

- s'il quitte l'unité de négociation c'est-à-dire le groupe visé pour quelque motif que ce soit, cette convention collective cesse de s'appliquer à son égard sauf s'il y a un rattachement exprès que l'on peut y retrouver. C'est souvent le cas lors du congédiement, de la mise à pied, de la procédure de rappel, etc.

- Par la durée limitée de la convention, il est entendu ou présumé l'être que l'ensemble de ses modalités sont susceptibles d'être modifiées, adaptées, améliorées ou restreintes à l'occasion de chaque renouvelle- 
ment. Cette règle s'applique sans égard à la qualité de sa disposition: bonne ou mauvaise, favorable ou défavorable au salarié, au syndicat ou à l'employeur.

À titre d'exemple, on doit accepter que le salarié mis à pied pour six (6) mois ne pourrait à son retour exiger l'indexation de son salaire alors qu'au cours du renouvellement de la convention collective, en cette même période, le syndicat dut y renoncer. Ainsi, lorsque ces deux personnes acceptèrent ces promotions de contremaître, ils devaient savoir, ou il faut le présumer au plan juridique, que le mode de calcul de l'ancienneté et que l'usage que l'on pouvait faire de ce moyen étaient susceptibles d'être modifiés à chaque renouvellement de convention collective. Dans la mesure où le retour de ces contremaîtres, à titre de salarié, survenait après la durée de la convention collective se terminant en 1975, il était inexacte de dire que:

... Les intimés promus contremaîtres ont quitté le giron du syndicat appelant alors que la convention collective assurait au cas de retour, qu'on leur compterait la durée de leur absence aux fins d'ancienneté. (juge Vallerand, p. 65)

La convention collective étant l'acte collectif des deux seuls parties signataires soit l'employeur et le syndicat accrédité et il leur est déjà suffisamment difficile d'en arriver à une entente, ces deux parties n'avaient nullement à consulter ou à prendre l'avis de ces deux contremaîtres pour effectuer quelques changements aux dispositions de leur convention collective de 1975. Il nous est donc difficile de comprendre que messieurs les juges Kaufman (à la page 65) et Chouinard retiennent la thèse opposée:

... ils ne pouvaient se voir priver d'un tel droit hors leur connaissance et sans leur consentement.

Remarquons que le Code du travail précise quand et comment le groupe de salariés actuel peut, à ce titre, participer à la décision de se lier par convention collective (art. 20.3 Ct). Même dans ce cadre, il s'agit d'une participation collective et non individuelle.

Selon les notes des trois juges de la Cour d'appel, il faudrait comprendre que les modalités conventionnelles relatives au cumul de l'ancienneté furent, en quelque sorte, intégrées aux contrats individuels de travail de ces contremaîtres et qu'elles ne pouvaient dorénavant être modifiées par les seuls auteurs de la convention collective et sans le consentement de ces contremaîtres. Cette approche est tout à fait contraire à l'économie même du droit collectif du travail et va à l'encontre de tout l'enseignement jurisprudentiel de la Cour suprême du Canada. Pour étayer cette double assertion, nous nous limitons à ce bref rappel:

- Parce qu'elle est à durée limitée (art. $65 C t$ ), on ne peut prolonger les effets de la convention collective autrement que par disposition spéciale et c'est ainsi que le législateur lui-même agit lorsqu'il entend imposer pareille prolongation. En effet, n'est-ce pas l'objet des articles 45, 59, 100.10 du Code du travail et de l'article 3 de la Loi sur les régimes supplémentaires de rente etc.;

- Combien de fois la Cour suprême du Canada a-t-elle reconnu l'autorité de la convention collective sur le contrat individuel de travail et affirmé 
ou souligné que cette convention était l'oeuvre exclusive de l'employeur et du syndicat accrédité au-delà des desiderata individuels des salariés? À cette fin, nous pourrions relire avec avantage ces seules décisions:

- Syndicat catholique des employés de magasin de Québec Inc. c. Compagnie Paquet Ltée, 1959 RCS 206;

- The Hamilton Street Railway Co. c. Northcott, 1967 RCS 3;

- McGavin Toastmaster c. Ainscough (1976) 1 RCS 918;

- General Motors of Canada Ltd c. Brunet et al., (1977) 2 RCS 537;

- Shell Canada Ltd. c. Travailleurs Unis du pétrole du Canada, 1982 RCS 181 .

Certes la durée limitée de la convention collective est importante pour permettre, s'il y a lieu, l'arrivée d'un autre syndicat ou pour provoquer un contrôle de la représentativité du syndicat (art. 22 et $41 \mathrm{Ct}$ ). Mais, cette limite temporelle permet également d'ajuster les conditions de travail à la conjoncture, de les mouler aux besoins mutuels et respectifs des parties par l'effet de la négociation, de la recherche de compromis à intervale assez régulier. À cette fin, 1'art. $62 \mathrm{Ct}$ reconnaît aux parties une grande liberté de manoeuvre: «La convention collective peut contenir toute disposition relative aux conditions de travail qui n'est pas contraire à l'ordre public ni prohibée par la loi». Si ces parties devaient être enclavées par les droits individuels résultant de la convention précédente et maintenant prolongés dans le temps par le contrat individuel des salariés qui en sont exclus, il leur serait encore plus difficile de conclure sinon impossible. Et s'il devait en être ainsi, alors des salariés exclus du groupe à la suite d'une promotion pourraient avoir plus de droits que ceux qui n'ont pas encore accepté de telles promotions! On remettrait ainsi en cause l'économie même du régime des rapports collectifs en créant de nouvelles inégalités entre salariés et en provoquant d'autres sources de conflit lorsqu'il s'agira de donner un sens pratique à l'ancienneté.

La Cour d'appel ne poussa pas d'ailleurs très loin ce raisonnement de la survie des droits subjectifs résultant de conventions collectives expirées par le biais du contrat individuel de travail. En effet, le juge Vallerand ne conclut-il pas en disant que «... il faut résoudre le problème d'applicabilité... en faveur de la première des deux conventions qui nous intéressent»! (p. 67) À notre avis, une seule et non deux conventions collectives pouvait juridiquement intéresser la Cour d'appel puisque celle expirée en 1975 était depuis longtemps disparue et qu'elle ne pouvait renaître ou se prolonger que par la volonté des parties et dans cette seule mesure, sauf si la loi en décide autrement, comme nous l'avons déjà souligné. Partant de cette approche de la survivance aussi partielle soit-elle de la convention collective de 1975, il est évident qu'il devenait difficile d'écrire des notes qui auraient pu être convaincantes, cohérentes et étayées par les dispositions du Code du travail et la jurisprudence de la Cour suprême du Canada. Ayant ainsi tranché l'affaire dite de l'applicabilité, le juge Vallerand s'en remet pour l'avenir à la compétence de «... l'arbitre de disposer de tout différend éventuel entre les parties» (page 67). Notons encore qu'il ne pouvait s'agir de différend mais bien de griefs et que tel que décidé maintenant par la Cour d'appel, les arbitres éventuellement saisis de ces griefs rencontreront quel- 
ques difficultés certaines à faire le point entre ce jugement et les droits qui peuvent résulter de l'actuelle convention collective. Imaginons, à titre d'hypothèse, que ces deux contremaîtres sont rétrogradés ou mis à pied et entendent se prévaloir de leur ancienneté pour supplanter quelques autres salariés? L'arbitre saisi du grief que pourrait provoquer cette situation aurait à son tour bien du mal à rédiger une sentence qui puisse être cohérente et aussi respectueuse de ce jugement de la Cour d'appel. Au-delà des grands principes de droit et des techniques juridiques d'interprétation, l'ancienneté telle qu'aménagée en une convention collective a un sens et une portée pratique tant pour les salariés que pour l'employeur. Analysons maintenant cette décision sous cet angle.

\section{L'ancienneté}

L'ancienneté est principalement un critère commode pour départager les salariés, pour effectuer des choix, pour établir un ordre de priorité entre eux. Elle confère d'abord un droit relatif, c'est-à-dire qui se conçoit et s'évalue qu'en rapport aux autres et dans un milieu donné et cela, contrairement à certains autres droits plus directement et isolément collés à leur titulaire: le salaire, le congé, etc. C'est ainsi que l'ancienneté établit l'ordre à suivre pour effectuer quelques mouvements de personnel: promotion, licenciement, prise de vacances, etc. Tous les salariés du milieu savent ainsi à quoi s'en tenir et, si les résultats ne sont pas nécessairement toujours heureux, ce critère vaut souvent mieux que la seule appréciation discrétionnaire de l'employeur notamment lorsqu'il est conjugué à d'autres facteurs pertinents et connus pour effectuer les choix nécessaires. Cette dimension de l'ancienneté semble n'avoir aucunement été retenue par la Cour d'appel qui considère ce droit à l'ancienneté comme s'il s'agissait d'un droit strictement subjectif rattaché à la seule personne des contremaîtres. Il y a d'ailleurs une contradiction flagrante entre cette approche individualiste et le qualificatif employé par le juge Vallerand «d'état syndical». Ce jugement de la Cour d'appel qui retient deux modes de calcul de l'ancienneté (celui valable pour les deux contremaîtres et celui applicable aux autres salariés), pourrait produire des effets opposés à ceux généralement recherchés par le critère de l'ancienneté. En effet, selon la convention collective actuelle, tel salarié peut légitimement croire qu'il détenait la priorité de retour au travail alors qu'il devrait céder sa place à l'un de ces deux contremaîtres rétrogradés ou mis à pied, etc. Il n'y a pas mieux pour engendrer frustration, insécurité, disparité et conflit au sein même du groupe de salariés et avec l'employeur et les autres contremaîtres. Cette dimension collective et celle de la relativité de ce droit et de sa finalité pratique semble avoir été ignorée! Pourtant, nous le répétons, ce mode de calcul de l'ancienneté établi à la convention collective de 1974 résultait d'une entente entre l'employeur et le groupe de salariés représenté par le syndicat tout comme d'ailleurs la règle conventionnelle établie en 1980. Pourquoi la première serait-elle plus permanente que la dernière alors qu'en ces deux cas, elles proviennent de la même source, en application du même processus et qu'elles sont engendrées en un même acte qui est, en chaque cas, d'une durée limitée? S'il fallait poursuivre jusqu'au bout la logique de la Cour d'appel en cette affaire, ne nous faudrait-il pas soutenir que ces deux contremaîtres pourraient aussi exiger de l'employeur 
de maintenir l'indexation directe de leur salaire depuis leur retour à titre de salariés, dans l'hypothèse où la convention collective applicable lors de leur nomination la leur accordait etc.? Un tel développement, poussé par l'imagination, illustrerait que ni les intérêts de l'employeur et ni ceux de la collectivité des salariés profiteraient de cette situation parce que les données basiques du régime des rapports collectifs du travail en seraient faussées.

Pour conclure, il nous faut simplement souhaiter que la voie du jugement déclaratoire utilisée en vertu de la théorie de «l'applicabilité» ne devienne pas la nouvelle avenue permettant et facilitant à nouveau l'intervention des tribunaux judiciaires maintenant que la voie de l'évocation sous prétexte de «la mauvaise question» ou de la conclusion «déraisonnable» fut quelque peu refermée par la Cour suprême du Canada.

\section{NEW ZEALAND JOURNAL OF INDUSTRIAL RELATIONS}

(ISSN 01100637 )

Editor: David F. Smith. Department of Business Administration. Victoria University of Wellington. Private Bag. Wellington. New Zealand.

AUGUST 1985 - Volume 10 No. 2

\section{ARTICLES}

Incomes policy and the 1985-1986 wage Jonathan Boston round: from non-market failure to market failure?

Maori pakeha differentials in incomes and

Peter Brosnan hours worked: a study of sample data for selected occupations

The earliest origins and suppression of

Kevin Hince trade unionism in the Fiji Islands

\section{COMMENTARY}

The Government's role in industrial relations

\section{REVIEWS CHRONICLE}

Published 3 times a year (April. August and December) by the Neu Fealand Invilute of Industrial Relations Research Inc.

Subscriptions: (Post paid)

\begin{tabular}{|c|c|c|c|}
\hline $\begin{array}{l}\text { Companies } \\
\text { and } \\
\text { Institutions }\end{array}$ & Personal & $\begin{array}{c}\text { Low Incomes } \\
\text { and Fill-time } \\
\text { Situdent }\end{array}$ & $\begin{array}{c}\text { Single Copies } \\
\text { and } \\
\text { Back-Issues }\end{array}$ \\
\hline 536 & $\$ 25$ & $\$ 12$ & $\$ 12$ \\
\hline SA27 & $\$ A 20$ & $\triangle A \mid 0$ & SA 9 \\
\hline SUS:7 & SUS?O & sus 10 & SUS 9 \\
\hline fStglK & EStgl2 & EStg6 & EStg6 \\
\hline
\end{tabular}

New Zealand

Ausiralia and South Paciinc

Nonh America and Asi.

Other countries

fStgls

fStgl?

EStg6

Orders: The Business Manager. New Zealand Journal of Industrial Relations. Box 28093. Kelburn. Wellington. New Zealand. 